SHORT REPORT

\title{
A randomised comparison of resuscitation with an anaesthetic rebreathing circuit or an infant ventilator in very preterm infants
}

\author{
J Menakaya, C Andersen, D Chirla, R Wolfe, A Watkins
}

Arch Dis Child Fetal Neonatal Ed 2004;89:F494-F496. doi: 10.1136/adc.2003.033340

Twenty four preterm infants ( $<27$ weeks gestation) were randomised to resuscitation with an anaesthetic rebreathing circuit or a ventilator with volume guarantee. There was no difference in weight or gestation between the groups. End expiratory pressures were higher and less variable with the ventilator. This pilot study shows that very preterm infants can be safely and effectively resuscitated using a ventilator.

A nimal studies suggest that the combination of excessive pressure and tidal volume can injure the lung. Few studies have documented respiratory mechanics in the first breaths, particularly in very preterm infants. ${ }^{2}$

The objective of this study was to measure respiratory mechanics during resuscitation and randomly compare an anaesthetic rebreathing circuit (hospital standard) with an infant ventilator with volume guarantee (Dräger Babylog 8000plus; Dräger Medizintechnik, Lübeck, Germany) for resuscitation in very preterm infants.

\section{METHODS}

Inborn singletons of 24-27 weeks gestational age (GA) were eligible. Infants with congenital thoracic abnormalities were excluded. The study was approved by the institutional research and ethics committee, and written informed consent was obtained. Infants were randomised within GA strata before delivery.

\section{Standard anaesthetic rebreathing circuit (SC)}

Resuscitation with a $500 \mathrm{ml}$ rebreathing anaesthetic bag, $100 \%$ non-humidified oxygen (6 litres/min), manometer (initial blow off set to $30 \mathrm{~cm} \mathrm{H}_{2} \mathrm{O}$ ), and face mask.

\section{Infant ventilator with volume guarantee (DB)}

Resuscitation using a Dräger Babylog 8000plus (synchronised intermittent positive pressure ventilation, 7 litres/min; humidified 100\% oxygen; positive end expiratory pressure (PEEP), $8 \mathrm{~cm} \mathrm{H}_{2} \mathrm{O}$; heart rate 60 beats/min; inspiratory time $0.35 \mathrm{sec}-$ ond with volume guarantee) with air and oxygen cylinders and an uninterrupted power supply (UPS-SOLA 610). A peak inspiratory pressure (PIP) of $40 \mathrm{~cm} \mathrm{H}_{2} \mathrm{O}$ was used for the first five infants. ${ }^{3}$ This was reduced to $25 \mathrm{~cm} \mathrm{H}_{2} \mathrm{O}$ for the remaining infants, as lower PIP was sufficient. A tidal volume of $5 \mathrm{ml} / \mathrm{kg}$ was based on the mean weight for GA (historic data). A face mask was used initially. If the infant remained bradycardic (heart rate $<100$ beats/min) at three minutes, the anaesthetic rebreathing circuit was used.

A separate neonatal team was present at delivery. Infants were intubated at the discretion of the neonatologist. All intubated infants were given surfactant (Beractant Suspension; Abbott Australasia) after admission.

\section{Respiratory mechanics}

A Florian Neonatal Respiratory Monitor connected to a laptop computer and placed immediately distal to the face mask recorded respiratory mechanics data at five second intervals including: endotracheal tube leak (\%), PIP, PEEP, mean airway pressure, breath frequency, and expiratory tidal volume (eVt). Attending staff were blinded to these data.

An Ohio transport incubator was used for the SC group. The DB group were transported in the transport incubator with the study ventilator. Data was captured during transport.

After admission, infants were ventilated with a Dräger Babylog 8000 (synchronised intermittent positive pressure ventilation with volume guarantee, $4 \mathrm{ml} / \mathrm{kg}$; flow, 7 litres/ min humidified gas; PIP, $25 \mathrm{~cm} \mathrm{H}_{2} \mathrm{O}$; PEEP, $6 \mathrm{~cm} \mathrm{H} \mathrm{H}_{2} \mathrm{O}$; ventilator frequency 60 beats/min; inspiratory time 0.35 second). Adjustments were initially made to inspired oxygen concentration and set volume guarantee $\left(\mathrm{PCO}_{2} 6-7.3 \mathrm{kPa}\right.$, $\mathrm{pH}>7.20$ ). Arterial saturation was maintained at $85-95 \%$.

\section{Lung volume}

Radiographs were obtained after stabilisation (within four hours) and lung area measured. ${ }^{4}$ Subsequent radiographs were obtained as indicated and reported by a blinded radiologist. Emphasis was placed on evidence of pulmonary interstitial emphysema and pneumothorax.

\section{Clinical data}

Clinical risk index for babies (CRIB) score, ${ }^{5}$ arterial to alveolar (a:A) oxygen ratio, oxygen at 36 weeks of postconceptual age, worst head ultrasound, retinopathy of prematurity, and death were recorded.

\section{Sample size and statistical analysis}

Observed traces of PEEP, PIP, and eVt were summarised and compared between groups. Changes over time in a:A ratio were analysed with linear regression.

For $80 \%$ power, between 10 and 54 subjects per group were required to detect a relative difference of $20 \%$ in total lung volume.

\section{RESULTS}

Forty infants met the inclusion criteria; 24 were included (11 DB, 13 SC). Reasons for exclusion were: equipment unavailable (three), parents not approached (eight), declined consent (five). Table 1 shows infant characteristics. All characteristics were similar apart from a higher mean CRIB score in the SC group.

Abbreviations: CRIB, clinical risk index for babies; DB, Dräger Babylog 8000 plus; eVt, expiratory tidal volume; GA, gestational age; PEEP, positive end expiratory pressure; PIP, peak inspiratory pressure; SC, standard anaesthetic rebreathing circuit 
Table 1 Basic details of infants in two arms of a randomised trial of resuscitation: standard anaesthetic rebreathing circuit and Dräger Babylog

\begin{tabular}{|c|c|c|}
\hline Variable & $\begin{array}{l}\text { Standard } \\
\text { anaesthetic } \\
\text { rebreathing circuit }\end{array}$ & $\begin{array}{l}\text { Dräger } \\
\text { Babylog }\end{array}$ \\
\hline Female* $^{*}$ & $6(46 \%)$ & $5(45 \%)$ \\
\hline $\begin{array}{l}\text { Gestational age } \\
\text { (weeks) } \dagger\end{array}$ & $26(24-27)$ & $26(24-27)$ \\
\hline Birth weight $(g) \ddagger$ & 758 (408-1052) & 805 (510-1164) \\
\hline Antenatal steroids* & $13(100 \%)$ & $11(100 \%)$ \\
\hline \multicolumn{3}{|c|}{$\begin{array}{l}\text { *Values are number (\%). } \\
\text { †Values are median (range). } \\
\ddagger \text { Values are mean (range). }\end{array}$} \\
\hline
\end{tabular}

\section{Respiratory data}

Complete data from the time of birth to admission were available in 15 infants (7 DB, 8 SC; fig 1). The average recording time was similar. Nine had incomplete recordings. PEEP was higher and less variable for infants in the DB group (table 2). There were no significant differences between the groups in other variables.

\section{Outcomes}

The median time of surfactant administration was similar between groups. Mean a:A ratio was higher in DB infants; however, there was no significant difference between groups in change over time. Differences in mortality and airleak could have been chance findings. One infant had a grade 4 intraventricular haemorrhage. No infant had a positive blood culture. All were treated with antibiotics. Two infants assigned to the ventilator arm needed resuscitation with the standard circuit because of equipment malfunction (battery failure in one, ventilator tubing leakage the other).

\section{DISCUSSION}

This pilot study shows that the randomised design can be used to evaluate resuscitation of very preterm infants. In keeping with previous literature, infants resuscitated with the anaesthetic rebreathing circuit received variable inspiratory and end expiratory pressure. ${ }^{6}$

Although it is known that PEEP is important in the development and maintenance of lung volume, there is little information about the most appropriate level in infants during resuscitation. ${ }^{2}$ A pressure of $8 \mathrm{~cm} \mathrm{H}_{2} \mathrm{O}$ was arbitrarily chosen to maximise clearance of lung fluid and quickly establish functional residual lung capacity. This study shows that the Dräger Babylog ventilator maintained more stable PEEP during resuscitation.

There was a substantial difference between the set and achieved tidal volumes for infants resuscitated with the Dräger ventilator. There are a number of reasons for this. Firstly, set tidal volume was based on GA rather than birth weight and may have overestimated tidal volume per kilogram in growth restricted infants. Secondly, a Florian Neonatal Respiratory Monitor was used to record respiratory
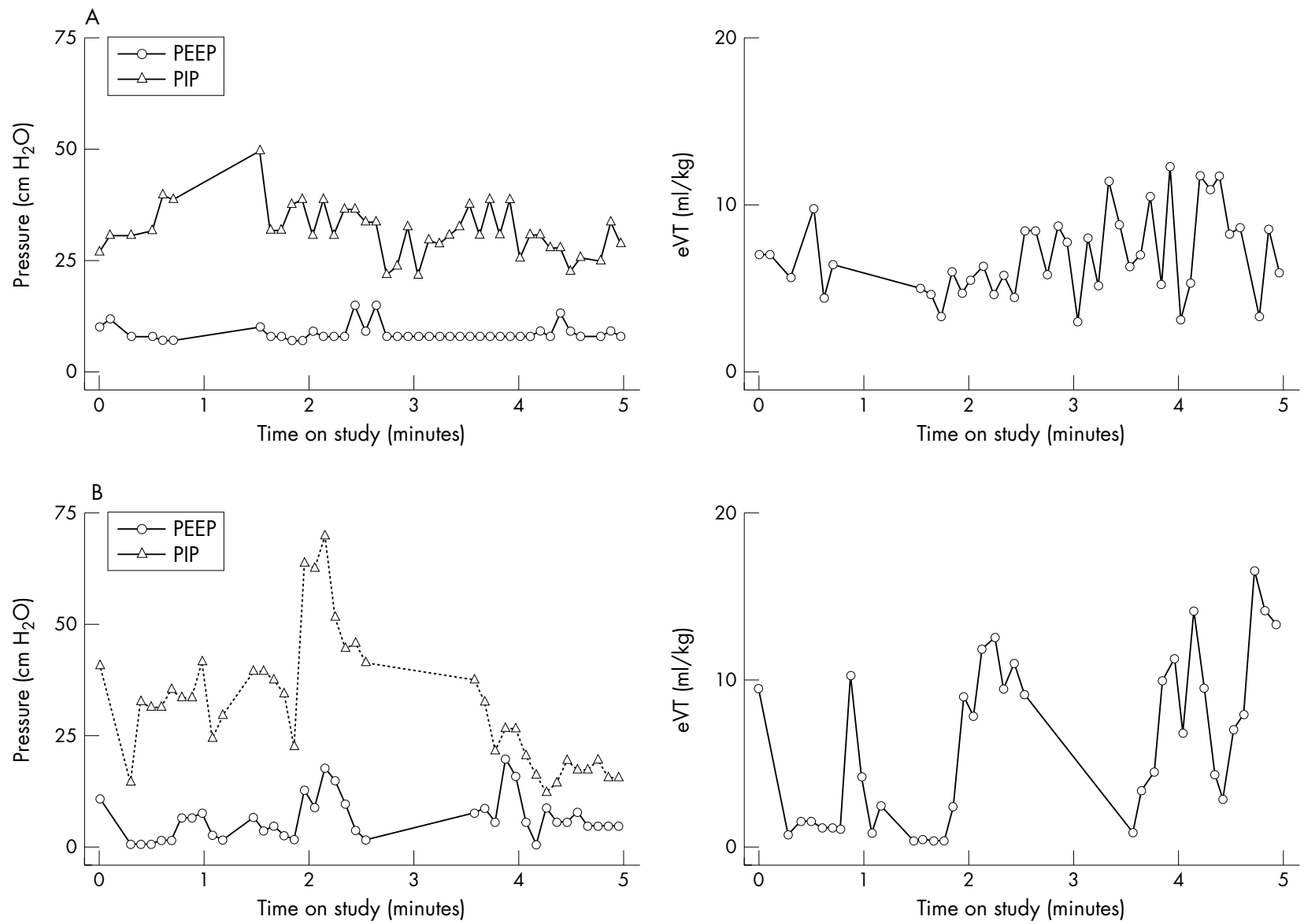

Figure 1 Infant respiratory mechanics data for the first five minutes. (A) An infant from the Dräger Babylog group (25.6 weeks gestation, weight $906 \mathrm{~g})$; (B) an infant from the standard anaesthetic rebreathing circuit group (27.4 weeks gestation, $1052 \mathrm{~g}$ ). PEEP, Positive end expiratory pressure; $\mathrm{PIP}$, peak inspiratory pressure; $\mathrm{eVt}$, expired tidal volume. 
Table 2 Respiratory mechanics and outcome data for infants in two arms of a randomised trial of resuscitation: standard anaesthetic rebreathing circuit and Dräger Babylog

\begin{tabular}{|c|c|c|c|}
\hline Variable & $\begin{array}{l}\text { Standard anaesthetic } \\
\text { rebreathing circuit } \\
\text { ( } n=13 \text { unless noted) }\end{array}$ & $\begin{array}{l}\text { Dräger Babylog } \\
\text { ( } n=11 \text { unless noted) }\end{array}$ & p Value \\
\hline PEEP (mean for time recorded; $\mathrm{cm} \mathrm{H}_{2} \mathrm{O}$ ) ${ }^{*}$ & $5.2(2.9-7.2)$ & $7.9(7.1-8.5)$ & $0.001 \cdot$ \\
\hline PEEP (CV for time recorded) $\dagger$ & $0.62(0.40-0.98)$ & $0.12(0.08-0.40)$ & $0.002 \S$ \\
\hline PIP (mean for time recorded; $\mathrm{cm} \mathrm{H}_{2} \mathrm{O}$ ) * & $24.5(14.6-36.8)$ & $24.0(16.8-31.7)$ & 0.88 \\
\hline PIP (CV for time recorded) $\dagger$ & $0.22(0.12-0.38)$ & $0.26(0.09-0.42)$ & $0.30 \S$ \\
\hline $\begin{array}{l}\text { eVt (mean for time recorded; } \mathrm{ml} / \mathrm{kg} \text { birth } \\
\text { weight/breath)* }\end{array}$ & $9.7(4.9-15.7)$ & $9.4(6.6-14.6)$ & $0.85^{\top}$ \\
\hline $\mathrm{eVt}(\mathrm{CV}$ for time recorded) $\dagger$ & $0.47(0.32-0.63)$ & $0.38(0.32-0.61)$ & $0.64 \S$ \\
\hline Age at intubation (minutes) $\dagger$ & $3(2-94+\dagger)$ & $2(1-140 \pm \ddagger)$ & \\
\hline $\mathrm{PCO}_{2}$ on admission $(\mathrm{kPa}) \dagger$ & $6.3(4.4-15.8)$ & $5.8(3.4-9.6)$ & $0.56 \S$ \\
\hline $\mathrm{FlO}_{2}$ on admission $(\%) \dagger$ & $32(21-100)$ & $40(21-100)$ & $0.84 \S$ \\
\hline Lung area $\left(\mathrm{mm}^{2} / \mathrm{kg}\right)^{*}$ & $1221(847-1895) n=12$ & $1152(725-1859) n=9$ & 0.62 \\
\hline Mortality $\ddagger$ & $4(31 \%)$ & $1(9 \%)$ & $0.33^{\star *}$ \\
\hline Oxygen at 36 weeks and/or death $\ddagger$ & $8(73 \%) n=11$ & $7(70 \%) n=10$ & $1.00 * *$ \\
\hline Airleak, PIE, or pneumothorax $\ddagger$ & $5(38 \%)$ & $1(9 \%)$ & $0.17^{* *}$ \\
\hline
\end{tabular}

"Time recorded" is the entire length of data recording. Mean time for infants on the standard anaesthetic rebreathing circuit $(\mathrm{SC})=20$ minutes, and the mean time for those on the Dräger Babylog (DB) $=19$ minutes. Summary of respiratory mechanics measurements for 15 infants (8 SC and 7 DB).

*Values are mean (range).

†Values are median (range).

$\ddagger$ Values are number (\%).

t†Maximum observed intubation time was seven minutes.

$\ddagger \ddagger$ Maximum observed intubation time was three minutes.

Two sample $t$ test.

$\S$ Wilcoxon rank sum test.

**Fisher's exact test.

PEEP, Positive end expiratory pressure; PIP, peak inspiratory pressure; PIE, pulmonary interstitial emphysema; eVt, expiratory tidal volume.

mechanics in both groups to avoid bias; however, the absolute volume measured is different from the Dräger Babylog using Dräger Babyview software (Dräger Medizintechnik) (unpublished observation). Thirdly, as the recording interval increased from the time of birth, the difference between set and achieved tidal volumes also increased. This is probably the result of spontaneously large tidal breaths. Finally, our own observations suggest that recurrent disconnection and sudden changes in compliance potentially result in recurrent overshoot in expired tidal volume.

The standard anaesthetic rebreathing circuit used in the study has variable PEEP. The effect size may well have been larger if the standard group had been resuscitated without PEEP as would happen with most commonly used self inflating circuits.

The small sample size is a limitation; however, this reflects the ethical and practical difficulties of conducting resuscitation research. Short term measures of successful resuscitation including oxygenation, $\mathrm{pH}$, and $\mathrm{PCO}_{2}$ were not different. Lung volume measured by an anteroposterior radiograph is pragmatic though imperfect, ${ }^{4}$ and, if a reduction in radiological injury were to be used as the primary outcome, a sample size of three or four times greater would be required.

This pilot study shows that respiratory variables can be recorded during resuscitation of very preterm infants and further that these infants can be safely resuscitated with an infant ventilator.

\section{ACKNOWLEDGEMENTS}

This study was supported by a generous donation by Perpetual Trustees, Aust. Ltd.

\section{Authors' affiliations}

J Menakaya, C Andersen, D Chirla, A Watkins, Department of Paediatrics, Mercy Hospital for Women, Melbourne, Australia R Wolfe, Department of Epidemiology and Preventive Medicine, Monash Medical School, Melbourne

Correspondence to: Dr Andersen, Department of Paediatrics, Mercy Hospital for Women, Clarendon St, East Melbourne, Victoria 3002, Australia; candersen@mercy.com.au

Accepted 26 April 2004

\section{REFERENCES}

1 Dreyfuss D, Soler P, Bassett $G$, et al. High inflation pressure pulmonary oedema. Respective effects of high airway pressure, high tidal volume and positive end expiratory pressure. Am Rev Respir Dis 1988;137:1159-64.

2 O'Donnell CP, Davis PG, Morley CJ. Resuscitation of premature infants: what are we doing wrong and can we do better? Biol Neonate 2003;84:76-82.

3 Milner AD. Resuscitation of the newborn. Arch Dis Child 1991;66:66-9.

4 Dimitriou G, Greenough A, Kavvadia V, et al. A radiographic method for assessing lung area in neonates. Br J Radiol 1999;72:335-8.

5 The International Neonatal Network. The CRIB (clinical risk index for babies) score: a tool for assessing initial neonatal risk and comparing performance of neonatal intensive care units. Lancet 1993;342:193-8.

6 Upton CJ, Milner AD. Endotracheal resuscitation of neonates using a rebreathing bag. Arch Dis Child 1991;66:39-42. 